\title{
SearchCal: a Virtual Observatory tool for searching calibrators in optical long-baseline interferometry
}

\section{The faint-object case}

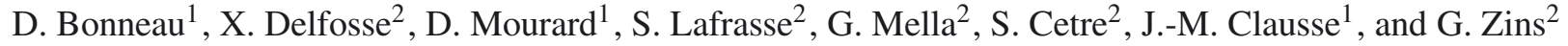 \\ ${ }^{1}$ Laboratoire Fizeau, UNS/OCA/CNRS UMR6525, Parc Valrose, 06108 Nice Cedex 2, France \\ e-mail: Daniel. Bonneau@oca.eu \\ 2 UJF-Grenoble 1/CNRS-INSU, Institut de Planétologie et d'Astrophysique de Grenoble (IPAG) UMR 5274, 38041 Grenoble, France
}

Received 1 June 2010 / Accepted 19 August 2011

\begin{abstract}
Context. In long-baseline interferometry, images or astrophysical parameters are obtained from the raw fringe contrast after a careful calibration process. We have already developed the software (SearchCal) to select suitable bright calibration stars $(V \leq 10 ; K \leq 5.0)$ for obtaining the ultimate precision of current interferometric instruments like the VLTI. With the expected gain in sensitivity of AMBER and PRIMA on the VLTI, the need for fainter calibrators should now be adressed.

Aims. We present a new version of SearchCal dedicated to the creation of an evolving catalog of stars suitable as calibrators with $K$ magnitude $>5$ around the scientific target.

Methods. Star catalogs available at the CDS are searched via web requests and provide the useful astrometric and photometric informations for selecting calibrators. The missing photometries are computed with an accuracy of about $0.1 \mathrm{mag}$. The stellar angular diameter is estimated with a precision of about $10 \%$ through newly determined surface-brightness versus color-index relations based on the $I, J, H$ and $K$ magnitudes. For each star the squared visibility is computed taking into account the central wavelength and the maximum baseline of the predicted observations.

Results. The version of SearchCal for faint objects that allows to find calibrators for interferometric observations up to $K \sim 15$ is available as a web service at the address: http://www. jmmc. fr/searchcal
\end{abstract}

Key words. stars: fundamental parameters - techniques: interferometric - catalogs - techniques: high angular resolution astronomical databases: miscellaneous

\section{Introduction}

Optical interferometers have proved to be powerful tools for obtaining spatially resolved images or for determining the size and morphology of stellar objects. The increase in sensitivity of these instruments allows one to observe much fainter objects which provides access to new fields of astrophysical research.

Following the power rise of the Very Large Telescope Interferometer (VLTI), the AMBER instrument is expected to reach the coherent limiting magnitude close to $K \simeq 9.0$ (Petrov et al. 2007). Moreover, the PRIMA instrument will be operational soon on the VLTI (Delplancke et al. 2006). It will require the use of astrometric reference stars brighter than $K \simeq 16$ and located at less than $10^{\prime \prime}$ of the scientific target. This is mandatory for reaching the ultimate astrometric accuracy of 10 microarcsecond for the determination of exoplanet astrometric orbit. To correctly eliminate the effects of the atmospheric turbulence and of the instrumental instabilities in the data processing, calibrators are necessary to convert the fringe contrast measurements properly into the intrinsic visibility of the scientific target (van Belle \& van Belle 2005). Searching for faint calibrators is now a necessity, and the selection of suitable faint calibration stars is crucial to reach the ultimate precision of the interferometric measurements.

The Centre Jean-Marie Mariotti (JMMC) has decided to develop a new version of the calibrator's searching tool called SearchCal (Bonneau et al. 2006, hereafter Paper I). In the first version of SearchCal (the bright-object case), the limiting magnitude for the calibrators (typically $V \leq 10$ and $K \leq 5$ ) agrees with the sensitivity of the visible or near-infrared interferometers in operation at that time. Some adaptations to the method are necessary in the faint-object case.

For searching calibrators, two strategies are usually chosen: the dynamic approach adopted for SearchCal and the closedlist approach based on a catalog of selected calibrators (Mérand et al. 2005). This second approach is used in the ESO Calvin Tool but seems to be unmanageable when working with fainter calibrators $(K \geq 6)$ that are too numerous.

Because of the strong density of faint stars in the sky, it was decided to automatically estimate the research field to gather almost 50 stars in the first request. The stellar database search is now based on the use of the catalogs 2MASS All Sky Catalog of Point Sources (Skrutskie et al. 2003) and the DENIS data base (The Consortium Denis 2005), which provide the infrared photometric data in the suitable range of magnitudes. Then, new surface-brightness versus color-index relations, based only on the infrared photometric magnitudes, were determined for the calculation of the stellar photometric angular diameter.

The two main differences with the bright-object case are that in most cases, neither the parallax of the candidates nor the magnitudes in all photometric bands will be known. The effect of interstellar absorption on the determination of angular diameter determination cannot be completely corrected for faint stars for 
which the distance is generally unknown. Consequently we have investigated to what extent these can possibly be neglected.

The development of the faint object version of SearchCal was made within the same framework as described in Sect. 5 of Paper I. A web service has been implemented for the general use of SearchCal.

Section 2 presents the method adopted for creating a dynamical list of stars fulfilling the requirements of interferometric faint calibrators $(K>5)$. Section 3 describes the new CDS request's process for extracting the useful parameters from selected stellar catalogs and to sort out the initial list of possible faint calibration stars. Section 4 describes the calculations (angular diameter, visibility) adapted to the faint-object case. The limitation of SearchCal are presented in Sect. 5 and we discuss the statistics of the calibrators selected by SearchCal in the last section.

\section{The method}

The goal of the faint-object version of SearchCal is the creation of a dynamical catalog of calibration stars that exceeds the limitation in magnitude of the current version (see Paper I). The same general approach is followed: after selecting stars in the stellar database with a maximum of astrometric and spectrophotometric parameters, we calculate the visibility and its error after an estimation of the angular diameter and taking into account the maximum spatial frequency $\left(\frac{\boldsymbol{B}}{\lambda}\right)$ of the interferometric observation.

The "Virtual Observatory" techniques were adopted to extract the required astronomical information from a set of stellar catalogs available at the Centre de Données astronomiques de Strasbourg (CDS). This strategy appears to be the only one adapted to the handling of the large stellar catalogs that are required by the increase of the limiting magnitude of the interferometric facilities such as the VLTI or the Keck Interferometer (KI). The present version of the software is limited to longbaseline interferometric observations carried out in the near infrared ( $J, H$ and $K$ bands).

To minimize the effects of temporal and spatial variations of the seeing on the calibration process, a calibrator must be as close as possible to the scientific target. To be observable with the same instrumental configuration, the magnitude of the calibrator in the observing photometric band must be in a narrow range of value around the target magnitude. To limit the number of stars after the first request, the size of the field is automatically fixed to limit this number to about 50 stars. The Besançon model of population synthesis (Robin et al. 2003) is used to compute the value of the number of stars $N_{\text {star }}$ in a solid angle of 1 degree for the galactic position of the scientific target and for the $K$-magnitude range chosen for the calibrators. The radius (in arcminutes) of the research field hat will yield 50 stars is then simply defined by

$$
r=30 \times \sqrt{\frac{50}{N_{\text {star }}}} .
$$

A certain number of astronomical parameters must be known for each star. These parameters are given in Table 1.

We use the two most extended near-IR photometric catalogs (2MASS and DENIS) to extract the mandatory infrared $H, J, K$ and $I$ magnitudes. Indeed, the infrared magnitudes up to 14 appears to be impossible to retrieve from the photometric catalogs (mainly JP11 and CIO) used in the bright-object version of SearchCal. An online interface with the VizieR data
Table 1. Astronomical parameters for faint calibration stars.

\begin{tabular}{lc}
\hline \hline Mandatory parameters & \\
\hline Identifiers & 2MASS, DENIS, \\
& HIP, Tycho, \\
Coordinates & RD, DM numbers \\
Proper motion & pmRA, DEJ2000 \\
IR photometry & $I, J, H, K$ magnitudes \\
\hline Optional parameters & $l I I, b I I$ \\
\hline Galactic coordinates & $\pi_{\mathrm{HIP}}$ \\
Parallax & temperature and luminosity class \\
Spectral type & $U, B, V, R$ magnitudes \\
Vis photometry & measured \\
Angular diameter & variability and multiplicity flags, \\
Miscellaneous & radial velocity, rotational velocity \\
&
\end{tabular}

base (Ochsenbein et al. 2000) is used to extract astrometric and spectro-photometric parameters of the sources in the calculated field and to obtain the initial list of stars (see details in Sect. 3).

If available, the measured angular diameter is obtained through the data contained in the Catalog of High Angular Resolution Measurements (Richichi et al. 2005).

For each star of the initial list, calculations are made to estimate the photometric angular diameter and its associated precision using a surface-brightness method based on the magnitudes and color index (see Sect. 4.2). Then, the expected visibility and its error are computed for the maximum baseline and the central wavelength chosen by the user.

Considering only infrared observations forces us to determine new surface-brightness versus color-index relationships based only on $I, J, H$ and $K$ magnitudes (see Sect. 4.3).

As for the bright-object case, the list of possible calibrators is finally proposed to the user and the final choice can be made by changing the selection criteria: precision on the calibrator visibility, distance to the target, variability and multiplicity flags, magnitude range, spectral type and luminosity class (if known).

\section{The CDS search}

The choice of the catalogs has been made on the basis of their limiting magnitude which should be compatible with the magnitude range of the calibrators expected with the new version of SearchCal. Therefore the stellar parameters are taken from the following catalogs:

- II/246: The 2MASS all-sky survey Catalog of Point Sources (Skrutskie et al. 2003);

- B/denis: catalog The DENIS data base (The Consortium Denis 2005);

- I/280: All-sky Compiled Catalog of 2.5 million stars (Kharchenko 2001);

- I/284: The USNO-B1.0 Catalog (Monet et al. 2003);

- J/A+A/431/773/charm2: Catalog of High Angular Resolution Measurements (Richichi et al. 2005).

The request is based on the use of UCDs (unified content descriptor) defining the data labels and the intervals on the data values. Our strategy is based on two successive requests of the VizieR data base. 


\subsection{Primary request}

An online request to the CDS is initiated to obtain the initial list of stars. This request is made in the catalog " 2 microns all sky survey" (2MASS) selected with respect to the available photometry up to $J_{2 \text { mass }} \leq 15.8, H_{2 \text { mass }} \leq 15.1$ and $K_{2 \text { mass }} \leq 14.3$ and for the quality of the equatorial coordinates. The following search criteria are used:

- the position of the scientific target as center of the field of search;

- the calculated radius of the field;

- the user-defined range of the $K$ magnitude for the calibrators;

- the existence of an optical (visible) source associated to the infrared source.

The flag "opt", which indicates the presence of an optical source associated to the infrared source is used to select stars from the USNO-B1.0 or the Hipparcos-Tycho catalog for which we can obtain accurate astrometrical data (equatorial coordinates, proper motions and possibly parallax).

The output of this first sequence is a list of star coordinates with magnitude values as specified in the initial request.

\subsection{Secondary request}

On the basis of the previous list, the catalogs ASCC (I/280) and USNO (I/284) are searched to extract astronomical parameters of the associated optical source. The equatorial coordinates, the proper motions, the parallaxes, the spectral types, and the variability or multiplicity flags are extracted (when present) from the $I / 280$ and $I / 284$ catalogs. The identifiers are extracted from 2MASS, DENIS and I/280. The infrared photometry is taken from the 2MASS ( $J, H, K$ magnitudes) and DENIS ( $I$ magnitude) catalogs. The 2MASS photometry is converted into CIT system through the relation derived by Carpenter (2001):

$$
\begin{aligned}
& K_{\mathrm{CIT}}=K_{2 \text { mass }}+0.024 \\
& J_{\mathrm{CIT}}=0.947 J_{2 \text { mass }}+0.053 K_{2 \text { mass }}+0.036 \\
& H_{\text {CIT }}=0.975 H_{2 \text { mass }}+0.025 K_{2 \text { mass }}-0.004 .
\end{aligned}
$$

The photometric quality of the 2MASS data is guaranteed by the restrictive use of the Qflag = AAA, meaning that the source was observed with a $S / N \geq 10$. To avoid a non-significant value of the $I$ magnitude caused by the saturation of the photometric data, the I magnitude is not extracted from the DENIS catalog for stars with the image and source flag $I f l g=4$. The visible photometry comes from the catalogs $\mathrm{I} / 280$ (given $B$ and $V$ Johnson magnitudes) and $\mathrm{I} / 284$ (given $B$ and $R$ photographic magnitudes). The galactic coordinates are taken from the 2 MASS catalog. If available, the measured values of the angular diameter are extracted from the CHARM2 catalog.

\subsection{Final list of possible calibrators}

All results are then parsed and merged in a single array of stars with all available astronomical parameters. The catalogs are linked according to the equatorial coordinates if they are identical to within 1 arcsec. The final result is a single list containing stars with the required astronomical parameters.

\section{The calibrator parameters calculation}

In this section we explain how we estimate the angular diameters of the stars contained in the final list of the CDS requests.
This is necessary to calculate their visibilities for the selected interferometric configuration. In the faint-object case, however, we have provided two scenarii with or without a known stellar distance. We consider that the distance is known if the Hipparcos parallax $\pi_{\text {HIP }}$ is larger than 1.0 millisecond of arc and if the relative precision $\Delta \pi_{\mathrm{HIP}} / \pi_{\mathrm{HIP}}$ is smaller than 0.25 . In the first case (known parallax), the angular diameter, when not already measured, is calculated by using the photometry corrected from interstellar absorption and the surface brightness relations as described in Paper I. Otherwise (unknown parallax), the correction of the interstellar absorption is not possible and therefore the angular diameter is estimated with new photometric relationships as explained in Sect. 4.3. It is consequently necessary to complete the missing values in the previous requests.

\subsection{Rebuilding missing photometries}

The measured values of at least three of the $I, J, H$ and $K$ magnitudes are mandatory to compute the angular diameter of the calibrator but the knowledge of the complete $B, V, R, I, J, H, K$ photometry can be useful for the interferometric observation and housekeeping operations (fringe tracking for example). Accordingly, we chose to use the $J$ and $K$ magnitudes to calculate the eventually missing $B, V, R, I$ and $H$ magnitudes from the "spectral type - luminosity class - color" relationships tabulated for dwarfs, giants and supergiant stars.

To cover the widest possible range in spectral types and luminosity classes, we compiled different relationsfor the complete set of filter BVRIJHK, from Johnson (1966; Bessell 1979; Schmidt-Kaler 1982; Bessell \& Brett 1988; The et al. 1990; Leggett 1992; Wegner 1994). We adopted the CIT-Cousins photometric system according to our main source of infrared photometry (2MASS and DENIS catalogs). The photometry of (Johnson 1966; Wegner 1994; Bessell \& Brett 1988), are respectively in Johnson, Johnson-Glass and Bessell-Brett photometric system. They are converted in the CIT-Cousins photometric system in using the color transformation of (Bessell 1983; Bessell \& Brett 1988; Glass 1975). The relations used in SearchCal faintobject are listed in Table 2 and shown in Fig. 1. If the class of luminosity of the star is known, the corresponding table is used. If not, the table of the luminosity class for the dwarfs is used because the dwarfs are the most numerous stars in our magnitude range ( $K$ between 5 and 14). This could add an error of a few tenths of magnitude for giant stars (which could be more numerous in the bright part of our sample at low galactic latitude).

To test our "spectral type - luminosity class - color" relationships we used the 4000 stars of Ducati (2002). This catalog contains spectral type and photometric measurement. We computed an $\mathrm{O}-\mathrm{C}$ value between observationnal and reconstructed color from the relationships. The $\mathrm{O}-\mathrm{C}$ values are on the order of $0.1 \mathrm{mag}(\mathrm{rms})$ for the visible and $0.2 \mathrm{mag}(\mathrm{rms})$ for the infrared, ensuring a correct reconstruction of the photometry (see Figs. 2 and 3 ).

\subsection{Determination of the angular diameter}

The stellar angular diameter is usually unknown and a surfacebrightness versus color-index relation should be used to estimate its value from photometry. In the SearchCal faint-object case, the principle of calculation is the same as for the bright-object case (see Paper I) but the magnitudes and the color index used are different. We used 2MASS and DENIS photometries, which are close to the CIT-Cousins photometric system, which is why the 
Table 2. Spectral type - color relations used in SearchCal.

\begin{tabular}{|c|c|c|c|c|c|c|}
\hline \multirow{3}{*}{$\frac{\text { Color }}{B-V}$} & \multicolumn{2}{|r|}{ Dwarfs (class V) } & \multicolumn{2}{|r|}{ Giants (class III) } & \multicolumn{2}{|c|}{ Supergiants (class I) } \\
\hline & O5-B9 & Wegner $(1994)^{a}$ & O5-B9 & Wegner $(1994)^{a}$ & O5-B9 & Wegner $(1994)^{a}$ \\
\hline & A0-K7 & Schmidt-Kaler (1982) & $\mathrm{A} 0-\mathrm{K} 7$ & Schmidt-Kaler (1982) & A0-K7 & Schmidt-Kaler (1982) \\
\hline & M0-M8 & Leggett (1992) & M0-M8 & Bessell (1979) ${ }^{a}$ & M0-M5 & Johnson $(1966)^{a}$ \\
\hline \multirow{3}{*}{$V-R$} & O5-B9 & Wegner $(1994)^{a}$ & O5-B9 & Wegner $(1994)^{a}$ & O5-B9 & Wegner $(1994)^{a}$ \\
\hline & A0-K7 & Bessell (1979) ${ }^{a}$ & A0-K7 & Bessell (1979) ${ }^{a}$ & A0-M5 & Johnson $(1966)^{a}$ \\
\hline & M0-M8 & Leggett (1992) & M0-M8 & The et al. (1990) & & \\
\hline \multirow{3}{*}{$V-I$} & O5-B9 & Wegner $(1994)^{a}$ & O5-B9 & Wegner $(1994)^{a}$ & O5-B9 & Wegner $(1994)^{a}$ \\
\hline & A0-K7 & Bessell \& Brett $(1988)^{a}$ & A0-K7 & Bessell \& Brett (1988) ${ }^{a}$ & A0-M5 & Johnson $(1966)^{a}$ \\
\hline & M0-M8 & Leggett (1992) & M0-M8 & The et al. (1990) & & \\
\hline \multirow{3}{*}{$I-J$} & O5-B9 & Wegner (1994) ${ }^{a}$ & O5-B9 & Wegner $(1994)^{a}$ & O5-B9 & Wegner (1994) ${ }^{a}$ \\
\hline & $\mathrm{A} 0-\mathrm{K} 7$ & Johnson $(1966)^{a}$ & A0-M7 & Bessell \& Brett $(1988)^{a}$ & A5-M5 & Johnson $(1966)^{a}$ \\
\hline & M0-M8 & Leggett (1992) & & & & \\
\hline \multirow{3}{*}{$J-H$} & O5-B9 & Wegner (1994) ${ }^{a}$ & O5-B9 & Wegner $(1994)^{a}$ & O5-B9 & Wegner (1994) ${ }^{a}$ \\
\hline & $\mathrm{A} 0-\mathrm{K} 7$ & Bessell \& Brett $(1988)^{a}$ & A0-M7 & Bessell \& Brett $(1988)^{a}$ & & \\
\hline & M0-M8 & Leggett (1992) & & & & \\
\hline \multirow{3}{*}{$J-K$} & O5-B9 & Wegner $(1994)^{a}$ & O5-B9 & Wegner $(1994)^{a}$ & O5-B9 & Wegner $(1994)^{a}$ \\
\hline & $\mathrm{A} 0-\mathrm{K} 7$ & Bessell \& Brett (1988) & $\mathrm{A} 0-\mathrm{M} 7$ & Bessell \& Brett (1988) ${ }^{a}$ & A1-M5 & Johnson $(1966)^{a}$ \\
\hline & M0-M8 & Leggett (1992) & & & & \\
\hline
\end{tabular}

Notes. ${ }^{(a)}$ Photometry converted into Cousins-CIT.

calculations are made in this system. In the near infrared we used the $I, H, J$ and $K$ magnitudes of the CIT-Cousins photometric system associated with the color index $(I-J),(I-K),(J-H)$, $(J-K)$ and $(H-K)$.

The estimation of the limb-darkened angular diameter $\theta_{\mathrm{ld}}$ (in mas) of a star is given as a function of the color index $(C I)$ and its magnitude (mag) by

$\theta_{\mathrm{ld}}=\psi_{\mathrm{mag}}(C I) \times 9.306 \times 10^{-0.2 \mathrm{mag}}$

with

$\psi_{\text {mag }}(C I)=\Sigma_{k} a_{k} C I^{k}$.

For each star, a test of photometric coherence is performed by comparing the computed diameters with the different color in$\operatorname{dex}, \theta[I J], \theta[I K], \theta[V K], \theta[J K]$ and $\theta[H K]$. The star is rejected from the list if one value of the angular diameter differs by more than $2 \sigma$ from the mean value $\theta_{\text {mean }}$. If the star is retained, $\theta_{\text {mean }}$ is adopted as an estimate of the angular diameter. The relative uncertainty of the estimated diameter is discussed in next section.

\subsection{Surface-brightness relations}

To be useful, the $\psi_{\mathrm{mag}}(C I)$ relations must be almost independent of the stellar luminosity class, with small dispersion and based on the photometric bandpass of 2MASS/DENIS ( $I, J, H, K$ magnitudes). Our relation was determined by compiling the stellar diameter from interferometric measurements (corrected from limb-darkening) or lunar occultation from (Barnes et al. 1978; Ségransan et al. 2003; Mozurkewich et al. 2003; Kervella \& Fouqué 2008). To compensate the lesser number of main-sequence stars in interferometric measurement catalogs, we completed our compilation by eclipsing binaries (Andersen 1991) for which the stellar diameter is directly determined from the eclipse duration. Our sample is not meant to be exhaustive. It aims to collect measurements for stars that are distributed over a wide range of temperature and luminosity class.

The IJHK photometry is obtained from JP11 (Ducati 2002) for the bright stars of (Barnes et al. 1978; Mozurkewich et al. 2003; Kervella \& Fouqué 2008) and from Leggett (1992) for the M-dwarfs of Ségransan et al. (2003) and from Hipparcos (Perryman 1997, I-band) and 2MASS catalogs (Cutri et al. 2003, $J H K$-band) for the eclipsing binaries and for the faintest stars of Kervella \& Fouqué (2008). JP11 and 2MASS photometry are converted in the CIT-Cousins system by using the color relation derived from (Bessell 1983; Bessell \& Brett 1988; Carpenter 2001). Interstellar reddening is corrected by using $A_{\mathrm{V}}$ value given by (Barnes et al. 1978; Mozurkewich et al. 2003; Andersen 1991).

In Fig. 4 we plot $\psi_{x}$, for all the stars of our sample, defined as (see Delfosse \& Bonneau 2004; or Paper I):

$\psi_{X}=\frac{\theta_{\mathrm{ld}}}{9.306 \times 10^{-0.2 m_{X}}}$

or

$\psi_{X}=\frac{D_{*}}{10^{\left(5-M_{X} / 5\right)}}$,

(where $\theta_{\mathrm{ld}}$ is the limb-darkened angular diameter in mas; $D_{*}$ is the stellar diameter expressed in solar diameter and $m_{x}$ and $M_{x}$ are apparent and absolute magnitude in $X$-bandpass) as function of the different near-infrared color-indices. In some cases, the relationship covers only a limited domain in colors. The late stars, showing a wide dispersion of angular diameters for a given color, are not taken into account for certain relationships (see Fig. 4). The polynomial fit of these relations, used in SearchCal faintobject, and the color domain in which they are valid are given in Table 3.

The first source of uncertainty in the computation of the angular diameter is the variance of the calibration residuals (variance of the $(\mathrm{O}-\mathrm{C}))$. This variance includes the intrinsic dispersion of the relations, the error on the measured diameters, and the error on the photometry. For each of the five polynomial fits the difference between the measured and the computed angular diameters $(\mathrm{O}-\mathrm{C})$ are determined for the stars of (Mozurkewich et al. 2003; Kervella \& Fouqué 2008), they are plotted in Fig. 5 as function of the color index. The rms of the $(\mathrm{O}-\mathrm{C})$ are shown in Table 3.

All relations give an angular diameter with an accuracy better than $10 \%$, except for the $\psi_{H}(H-K)$ relation which ensures 
D. Bonneau et al.: SearchCal: the faint-object case
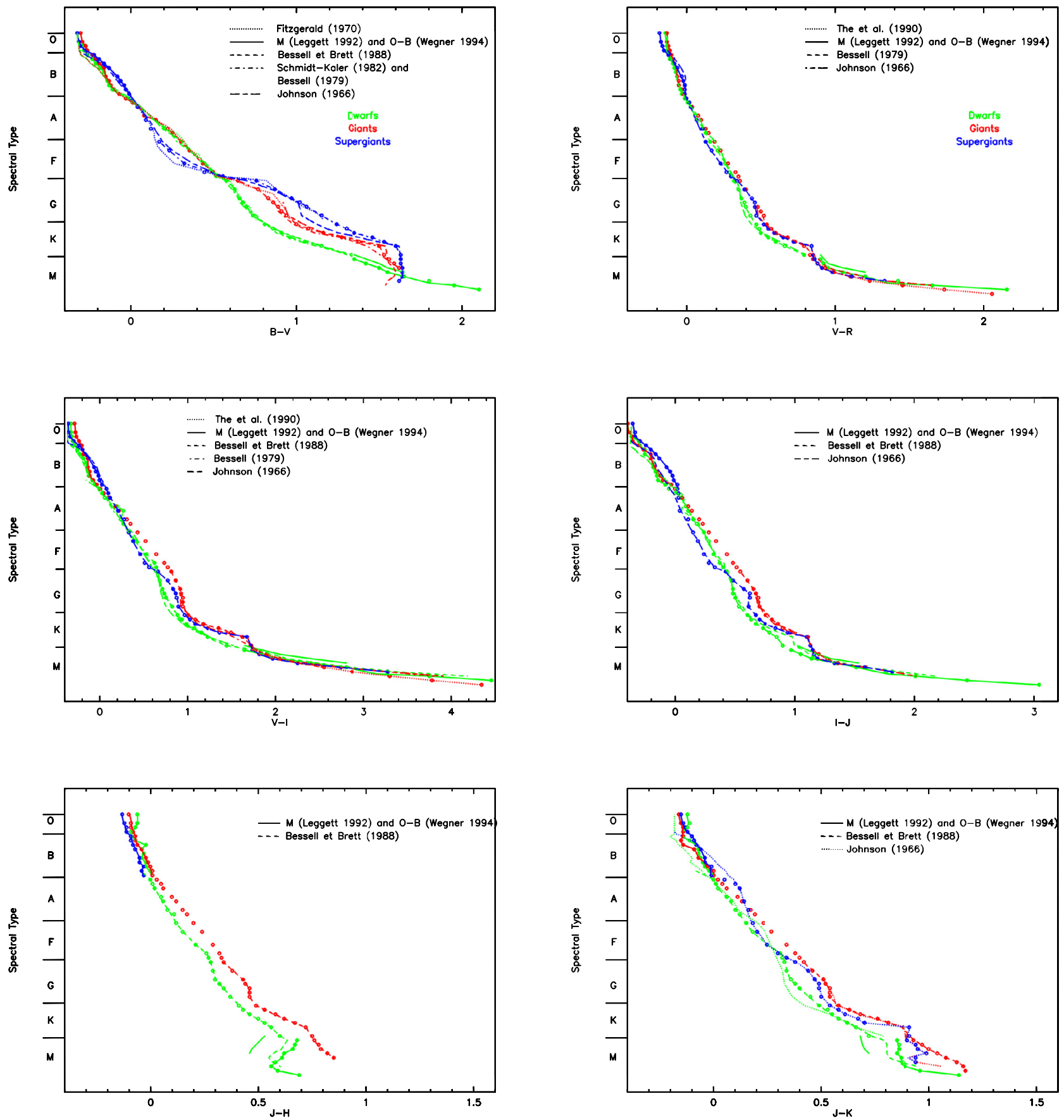

Fig. 1. Compilation of spectral type versus color relation in the litterature for luminosity class I (blue), III (red) and V (green). The adopted values of these relations in SearchCal faint-object are showed with the circle with the same color label for the three luminosity classes.

an accuracy of $\sim 13 \%$ that is yet tolerable for stars with a very small angular diameter. We chose these five relations to determine the stellar angular diameter in SearchCal to select for each of them the validity domain (in color index) in which the errors are stable.

\subsection{Calculation of the visibility}

We then calculated an estimate of the visibility for the central wavelength of the photometric band used for the observation. Considering the star as an uniform disk, the visibility $V_{\text {cal }}$ and its associated error $\Delta V_{\text {cal }}$ are computed as a function of the angular diameter $\theta_{\mathrm{ud}}$ (mas) and its error $\Delta \theta_{\mathrm{ud}}$ for the given instrumental configuration (wavelength $\lambda(\mathrm{nm})$ and maximum baseline $\left.B_{\max }(\mathrm{m})\right)$ :

$V_{\text {cal }}=\left|2 * \frac{J_{1}(x)}{x}\right|$

$\Delta V_{\mathrm{cal}}=2 * J_{2}(x) * \frac{\Delta \theta_{\mathrm{ud}}}{\theta_{\mathrm{ud}}}$

with $x=15.23 * \frac{B_{\max } * \theta_{\mathrm{ud}}}{\lambda}$.

To faint stars and given the typical values for the photometric angular diameter (typically $\theta_{\mathrm{ld}} \leq 1$ mas for $K>5.5$ ), the expected visibility bias induced by the use of $\theta_{\mathrm{ld}}$ instead of $\theta_{\mathrm{ud}}$ in Eq. (6) 

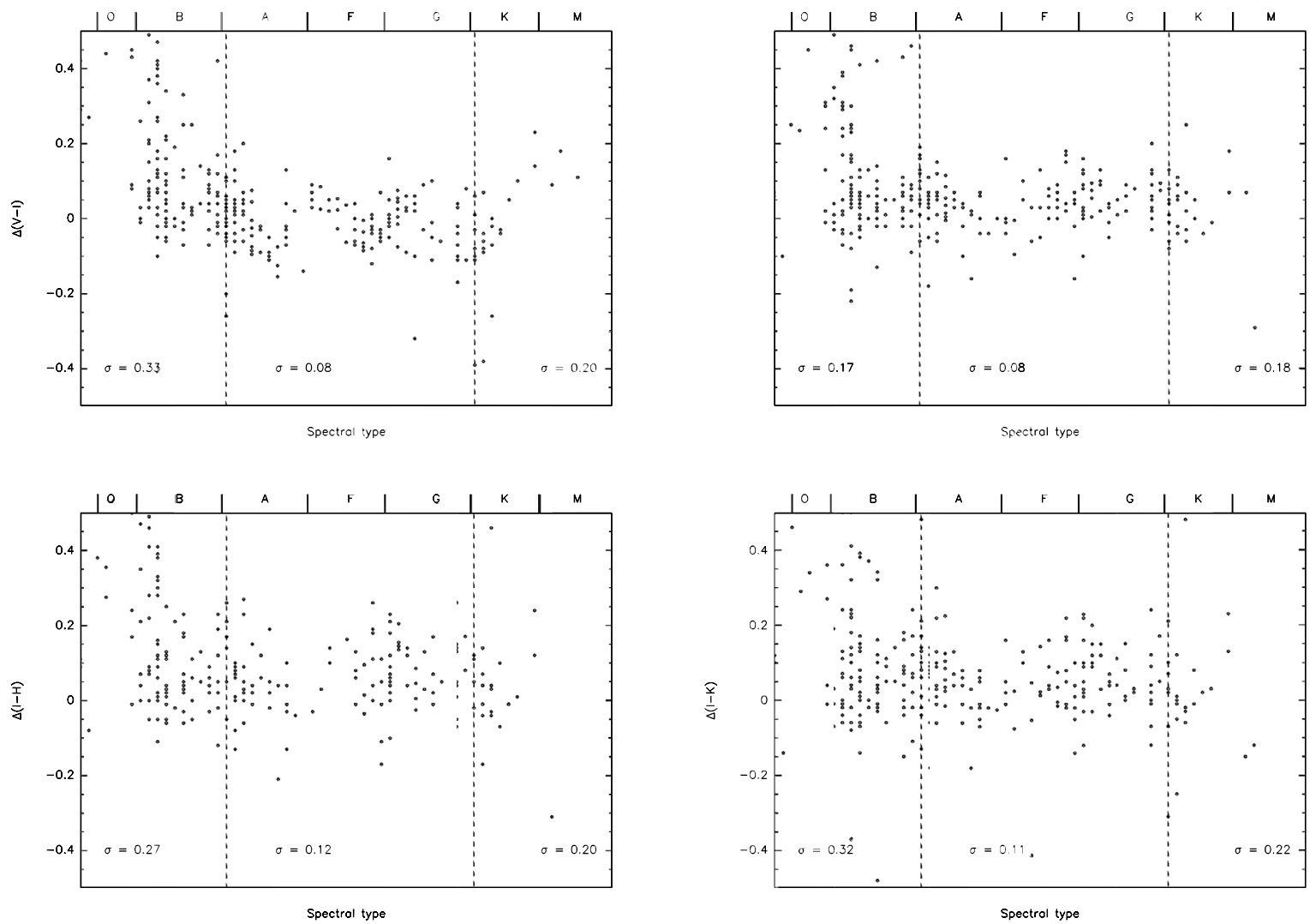

Fig. 2. (O-C) of the reconstructed colors for dwarf stars. We plot the difference between colors obtained from our "spectral type - luminosity class - color" relationships and from photometric measurement. Each circle corresponds to a star in the Ducati (2002) catalogs and with a know spectral type. The rms of the $(\mathrm{O}-\mathrm{C})$ are computed in three ranges of spectral type. We emphasize that the interstellar reddening is not corrected in the Ducati (2002) catalog, this is certainly the more important source of scattering for OB stars and M-giants (statistically more distant stars and therefore more subject to reddening).
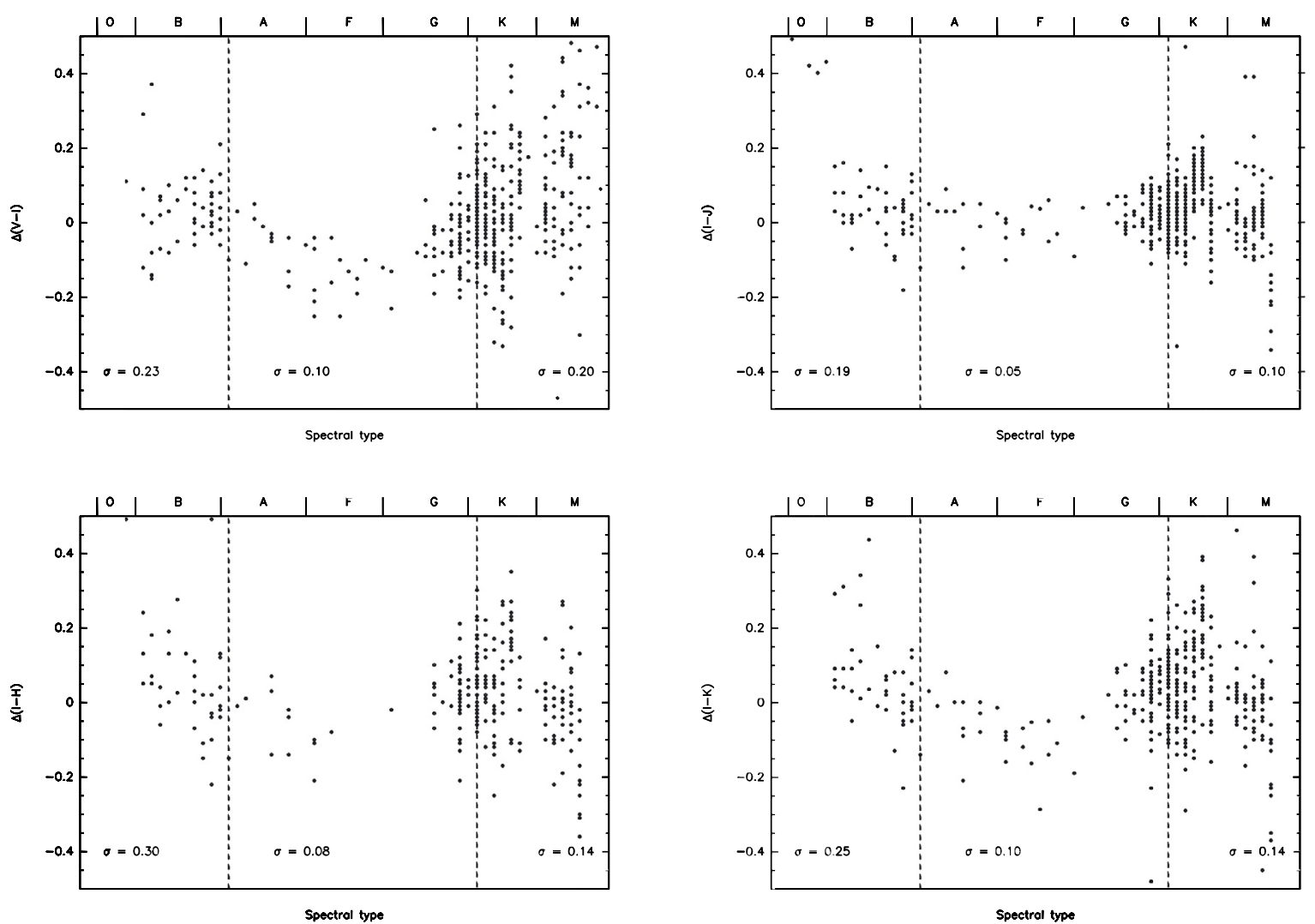

Fig. 3. Same as Fig. 2 but for giants stars. 

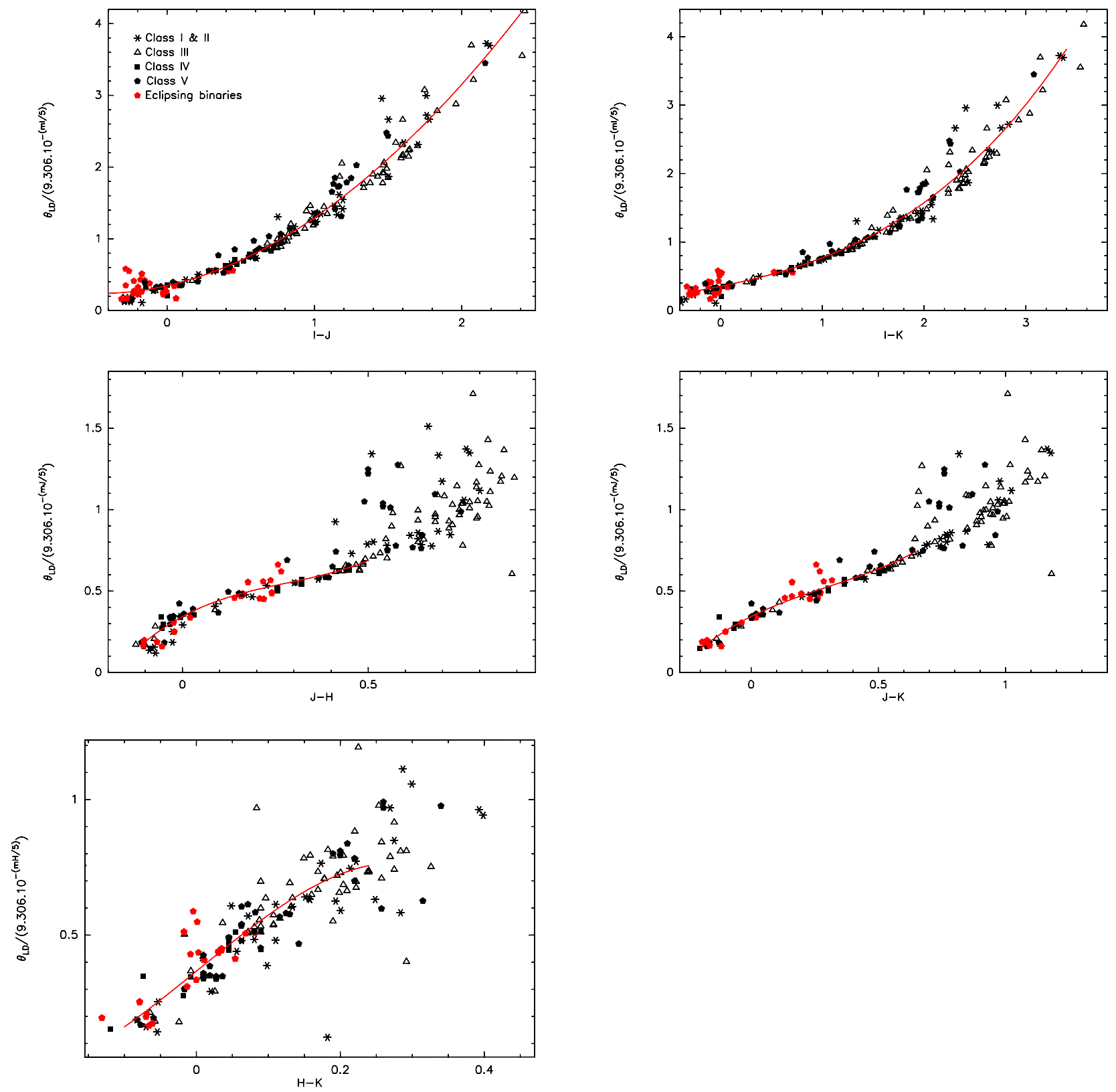

Fig. 4. The five surface-brightness versus color-index relations used in SearchCal for faint objects to determine the limb-darkened angular radius of calibrators. From left to right and top to bottom we show $\psi_{I}(I-J), \psi_{I}(I-K), \psi_{J}(J-H), \psi_{J}(J-K), \psi_{H}(H-K)$. The red line is our polynomial fit. Symbols for different luminosity classes: asterisk (super-giants I and bright giants II), open triangle (giants III), filled square (sub-giants IV), filled pentagon (dwarfs V). Data from direct angular diameter measurements are plotted in black and data from eclipsing binaries in red.

is always less than $5 \%$ in the $K$ band $(\lambda=2.2 \mu \mathrm{m})$ for baselines up to $150 \mathrm{~m}$ as shown in Paper I (see Fig. 5).

\subsection{Error on the calculated visibility}

The error on the calculated visibility is the result of three sources of uncertainties. The first, $\Delta V_{\text {phot }}$, comes from the uncertainty on the value of the computed angular diameter estimated from the calibration uncertainty of the surface-brightness relations as discussed in Sect. 4.3. The second, $\Delta V_{\mathrm{UD}}$, the visibility bias induced by the use of the limb-darkened photometric diameter instead of the uniform disk diameter is analyzed in Paper I. The third, $\Delta V_{\text {ism }}$, resulting from the photometric bias induced by the effect of the interstellar absorption, is studied in Sect. 5. For faint calibration stars with $\mathrm{Kmag} \geq 7$, the angular diameter is always $<0.25$ mas and for observations at $2.2 \mu \mathrm{m}$ with a baseline of up to $200 \mathrm{~m}$, this different contribution can be estimated to $\Delta V_{\text {phot }}< \pm 0.003, \Delta V_{\mathrm{UD}}<0.004$ and $\Delta V_{\text {ism }}<0.022$. It should be noted that the error on the computed visibility is dominated by the effect of the large-scale interstellar absorption.

\section{Effect of interstellar absorption}

In the majority of cases, the luminosity classes of potential calibrators are unknown and then the interstellar absorption in the line of sight of the stars cannot be estimated. Angular diameters are computed assuming $A_{V}=0$. If the absorption is significant this could have two effects:

- the star is bluer than observed and its angular diameter is in reality inferior to the computed one for the same apparent magnitude; 

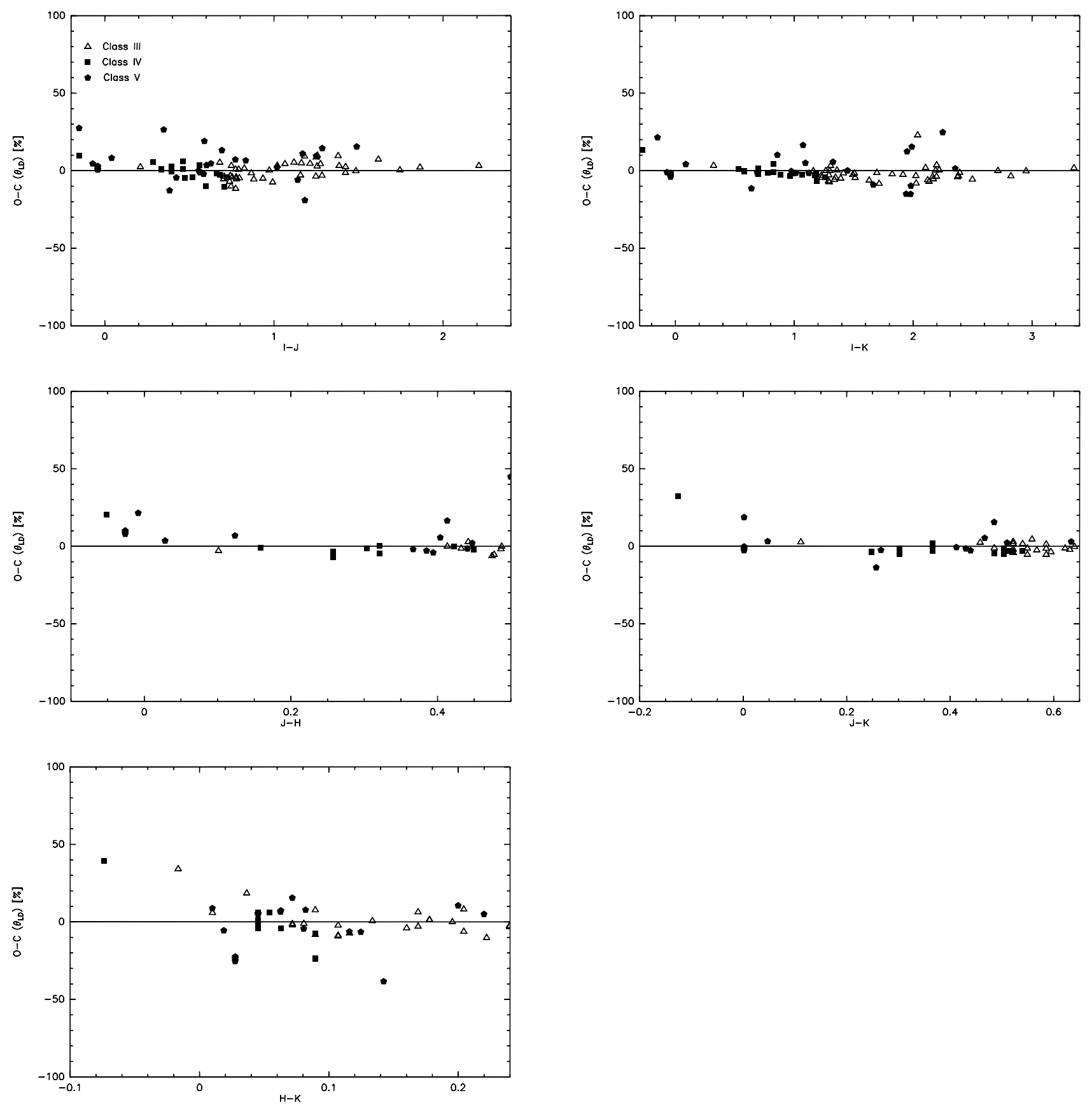

Fig. 5. Relative difference between observed angular diameters $\theta_{\mathrm{LD}}(\mathrm{obs})$ and computed ones $\theta$ (comp) with the five relations of Table 3 for the stars of Mozurkewich et al. (2003); Kervella \& Fouqué (2008). $\mathrm{O}-\mathrm{C}\left(\theta_{\mathrm{LD}}\right)=\left(\theta_{\mathrm{LD}}(\mathrm{obs})-\theta(\mathrm{comp})\right) / \theta_{\mathrm{LD}}(\mathrm{obs})$ are plotted in percent. The symbols are the same as for Fig. 4 .

- the star is brighter than observed and its angular diameter is in reality superior to the computed one for the same color.

The two effects work in opposite directions, but they do not necessarily cancel each other out. To estimate if neglecting the interstellar absorption could produce sensitive errors in the determination of the visibility in SearchCal faint-object, we have simulated its effect. Stars of luminosity class I, III, and V were generated in a grid of spectral type, distance, and position on the sky. The spectral type - color relationships of Table 2 were used to compute absolute magnitude and color of stars between A0 and late-M spectral type for the three luminosity classes. The stars were placed at distances between $10 \mathrm{pc}$ and $10 \mathrm{kpc}(d=$ $2^{n} \times 0.01 \mathrm{kpc}$ with $n$ between 1 and 10 to be regularly sampled in magnitude) and at a position along the galactic plane $\left(l_{\mathrm{II}}\right.$ between $0^{\circ}$ and $360^{\circ}$ and $b_{\mathrm{II}}$ between $-30^{\circ}$ and $+30^{\circ}$ with a step of $2.5^{\circ}$ ). Interstellar absorption at each position and distance are computed by using preferentially the $3 \mathrm{D}$ interstellar extinction distribution of Marshall et al. (2006) or that of Hakkila et al. (1997) for the positions and distances not available in the first paper. The effect of extinction in each photometric filter was computed by using the Fitzpatrick (1999) extinction laws. Angular diameter and visibility were then computed with and without extinction for two typical cases of baseline of $100 \mathrm{~m}$ and $200 \mathrm{~m}$ and for a central wavelength of $1.5 \mu \mathrm{m}$.

Figures 6 and 7 show the maximum value of these relative errors on visibility as a function of $l_{\mathrm{II}}$ and $b_{\mathrm{II}}$ computed for 
Table 3. Polynomial coefficient of the $\psi_{V}=\Sigma_{k} a_{k} C I^{k}$ relation for the five selected color indices.

\begin{tabular}{lcccccc}
\hline \hline Relat. & Validity domain & $a_{0}$ & $a_{1}$ & $a_{2}$ & $a_{3}$ & Accuracy \\
\hline$\psi_{I}(I-J)$ & {$[-0.4 ; 2.4]$} & 0.345406 & 0.463602 & 0.492598 & -0.010945 & $7.7 \%$ \\
$\psi_{I}(I-K)$ & {$[-0.3 ; 3.4]$} & 0.354057 & 0.302133 & 0.072303 & 0.040738 & $6.0 \%$ \\
$\psi_{J}(J-H)$ & {$[-0.1 ; 0.5]$} & 0.343007 & 1.246796 & -2.741362 & 3.250034 & $9.6 \%$ \\
$\psi_{J}(J-K)$ & {$[-0.2 ; 0.65]$} & 0.342905 & 0.780303 & -0.855117 & 0.928470 & $8.2 \%$ \\
$\psi_{H}(H-K)$ & {$[-0.1 ; 0.24]$} & 0.366284 & 2.159581 & 0.094470 & -9.658521 & $13.3 \%$ \\
\hline
\end{tabular}

Notes. Each relation is defined only in a given validity color domain. See the text and Fig. 5 for the accuracy determination.
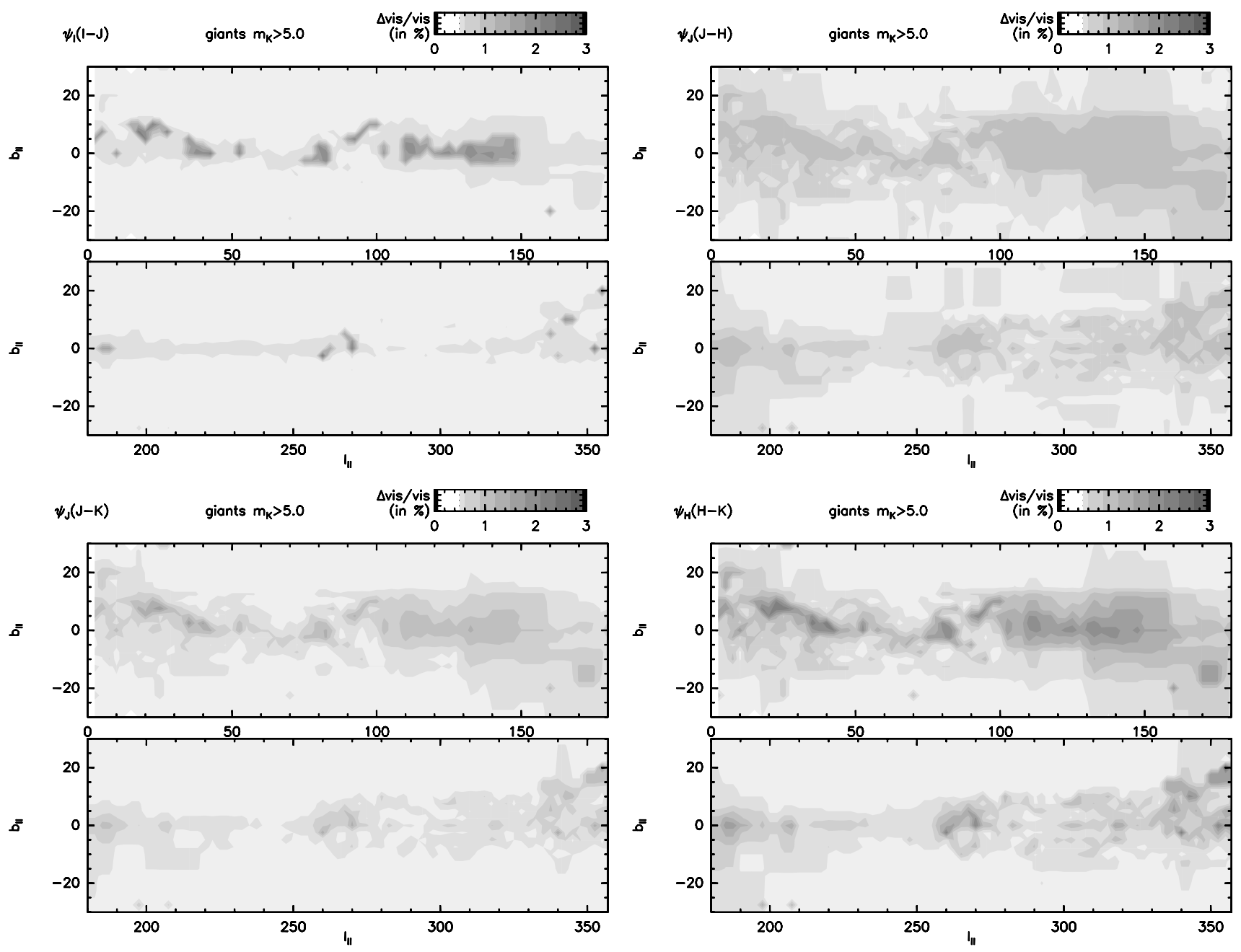

Fig. 6. Maps of maximum errors in the reconstruction of the visibility when the interstellar absorption is neglected. The maps are for giants stars fainter than $K=5$, a baseline of 100-m and computed with the surface-brightness relations $\psi_{I}(I-J), \psi_{J}(J-H), \psi_{J}(J-K)$ and $\psi_{H}(H-K)$.

four surface-brightness relations and for giants stars. The map for $\psi_{I}(I-K)$ is not reproduced but it shows a spatial behavior similar to that of $\psi_{I}(I-J)$ but with an error level two times lower.

Tables 4 and 5 present the maximum errors (integrated for all sky positions) computed with the five surface-brightness relations and for the three luminosity classes, the two baselines $(100$ and $200 \mathrm{~m}$ ) and for stars brighter than $K=5$ and $K=7$. For a $100 \mathrm{~m}$ baseline the errors are smaller than $3 \%$ in any sky position. For a $200 \mathrm{~m}$ baseline this $3 \%$ limit is reached for stars fainter than $K=7$. For calibrators selected for a baseline of $200 \mathrm{~m}$, with a magnitude close to $K=5$ and located in the galactic plane, the visibility estimated by SearchCal could be biased up to about $10 \%$.

In almost all cases the errors are small enough and we can conclude that neglecting the interstellar absorption is a valid assumption. One needs to be careful in case of red calibrators, close to $K=5$, located in the galactic plane and for baselines longer than $\sim 200-\mathrm{m}$.

For future interferometers with long baselines it will be necessary to reconsider this assumption. We also point out that our study has only considered the large-scale galactic absorption and not the very high absorption in stellar-formation regions. In these regions $A_{V}$ may rise to very high values at relatively close distances and therefore it will have a strong impact on the calculation of visibility.

\section{Summary and conclusion}

To analyze the results provided by SearchCal, we used the list of calibrators obtained on 16 reference fields defined during 

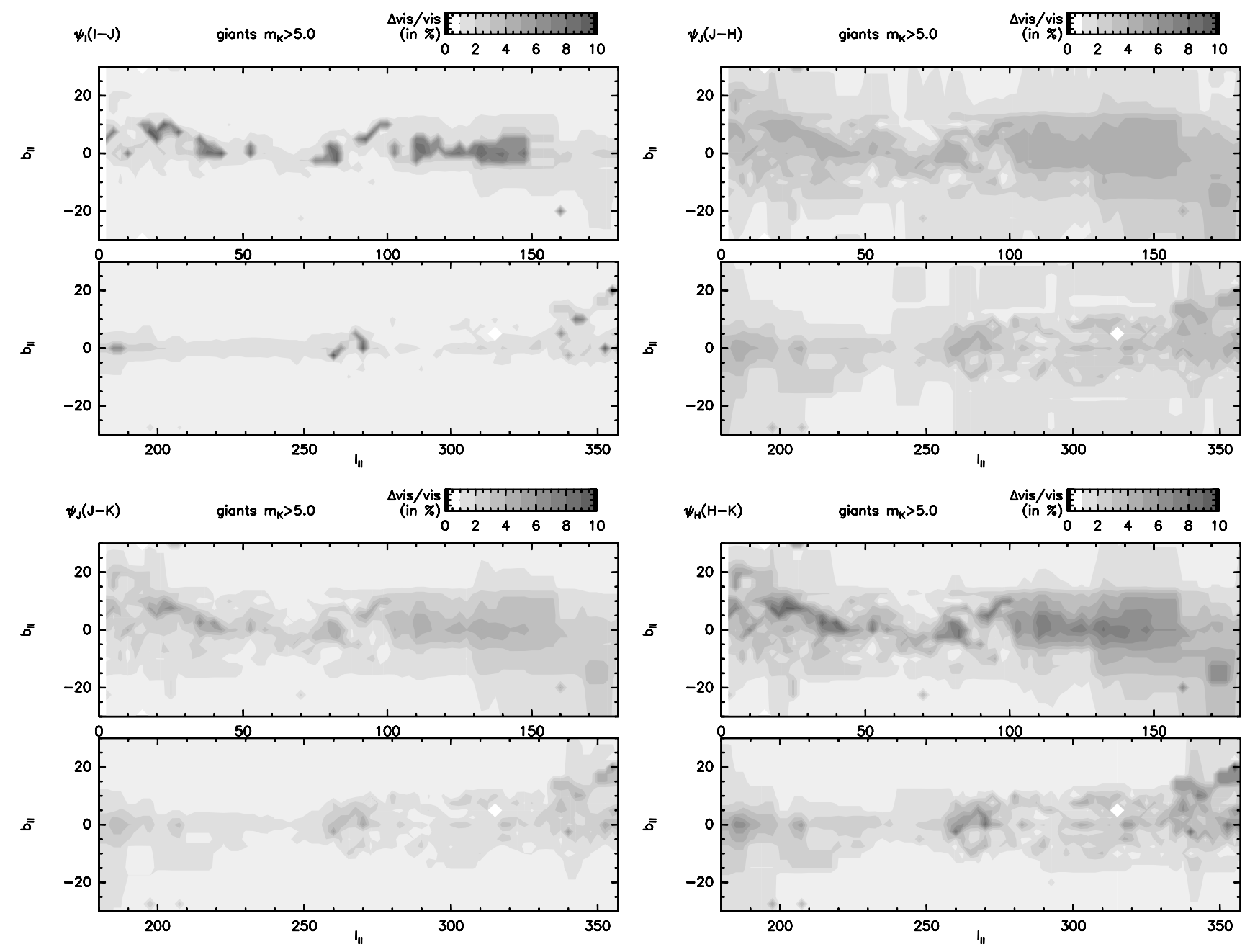

Fig. 7. Same figure than Fig. 6 for a baseline of 200-m.

Table 4. Maximum error on the visibility (integrated for all sky positions) when neglecting the interstellar absorptions for $K \geq 5.0$.

\begin{tabular}{lccccc}
\hline \hline & $(I-J)$ & $(I-K)$ & $(J-H)$ & $(J-K)$ & $(H-K)$ \\
\hline \multicolumn{5}{c}{$B=100 \mathrm{~m}$} \\
dwarfs & $0.7 \%$ & $0.7 \%$ & $1.0 \%$ & $1.7 \%$ & $2.6 \%$ \\
giants & $2.4 \%$ & $1.2 \%$ & $1.3 \%$ & $1.9 \%$ & $2.8 \%$ \\
supergiants & $2.1 \%$ & $2.5 \%$ & $1.6 \%$ & $2.7 \%$ & $2.7 \%$ \\
\hline \multicolumn{5}{c}{$B=200 \mathrm{~m}$} \\
dwarfs & $2.9 \%$ & $2.9 \%$ & $4.2 \%$ & $6.9 \%$ & $10.0 \%$ \\
giants & $10.0 \%$ & $5.0 \%$ & $5.5 \%$ & $7.5 \%$ & $11.0 \%$ \\
supergiants & $9.4 \%$ & $10.0 \%$ & $6.4 \%$ & $10.6 \%$ & $10.6 \%$ \\
\hline
\end{tabular}

the development phases. The galactic coordinates of these fields (radius 10 arcmin) are given in Table 6 as well as the number of stars returned as potential calibrators (diamOK) or rejected (diamKO). For this analysis, the stars were gathered into two categories corresponding to the fields of low galactic latitudes (4778 stars with $b<20 \mathrm{deg}$ ) or high galactic latitudes (2340 stars with $b \geq 20 \mathrm{deg}$ ).

The characterization of the stars selected as potential calibrators is illustrated by Fig. 8, which gives firstly the number of the calibrators found per squared-degree and per magnitude as a function of the $K$ magnitude and secondly the distribution of the calibrators as a function of the $(I-J)$ color index for high and low galactic latitudes. This figure gives an estimate of

Table 5. Maximum error on the visibility when neglecting the interstellar absorptions for $K \geq 7.0$.

\begin{tabular}{|c|c|c|c|c|c|}
\hline & $(I-J)$ & $(I-K)$ & $(J-H)$ & $(J-K)$ & $(H-K)$ \\
\hline \multicolumn{6}{|c|}{$B=100 \mathrm{~m}$} \\
\hline dwarfs & $0.2 \%$ & $0.1 \%$ & $0.3 \%$ & $0.4 \%$ & $0.5 \%$ \\
\hline giants & $0.3 \%$ & $0.2 \%$ & $0.5 \%$ & $0.5 \%$ & $0.6 \%$ \\
\hline supergiants & $0.3 \%$ & $0.4 \%$ & $0.2 \%$ & $0.3 \%$ & $0.2 \%$ \\
\hline \multicolumn{6}{|c|}{$B=200 \mathrm{~m}$} \\
\hline dwarfs & $0.8 \%$ & $0.6 \%$ & $1.1 \%$ & $1.5 \%$ & $1.9 \%$ \\
\hline giants & $1.4 \%$ & $0.9 \%$ & $2.2 \%$ & $2.1 \%$ & $2.3 \%$ \\
\hline supergiants & $1.2 \%$ & $1.9 \%$ & $0.8 \%$ & $1.4 \%$ & $0.8 \%$ \\
\hline
\end{tabular}

the number of calibrators expected to be found by SearchCal. For example, about a tenth of calibrators should be expected between $K=7$ and 8 in 1 square-degree at high galactic latitudes. It appears also that obtaining calibrators with $I-J$ color between 0.4 and 1.1 (or spectral type between G0 and early-M) is easy even very close to the scientific target; on the other hand looking for earlier or late calibrators will lead to larger angular distances especially for strong constraints on the magnitude of the object.

Approximately $50 \%$ of the stars from the initial list produced by the CDS requests are kept after the test of coherence on the photometric diameters. The majority of the rejected stars are reddened ones that lead to incoherent photometry. Figure 9 shows 

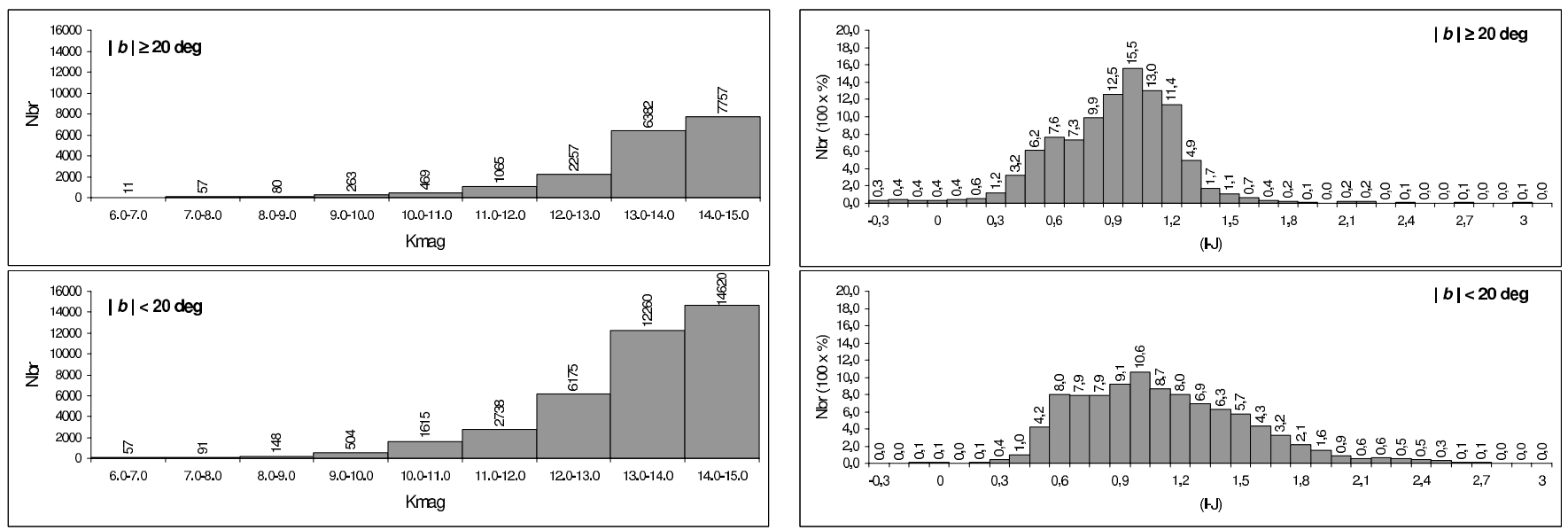

Fig. 8. Left: number of potential calibrators (per square degree) as function of the $K$ magnitude. Right: distribution (in $100 \mathrm{x} \%)$ of the $(I-J)$ color for stars of high and low galactic latitude.
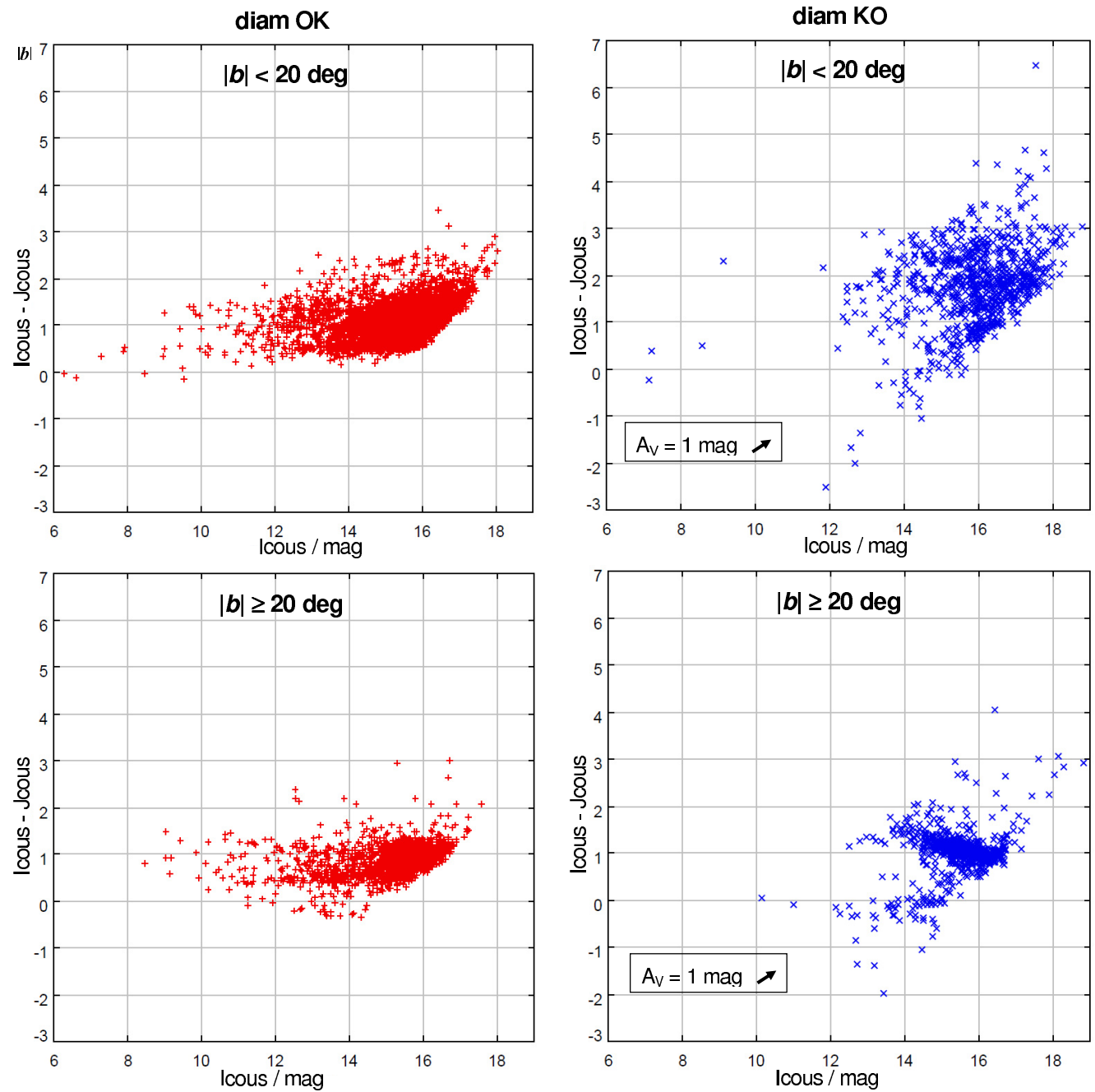

Fig. 9. Color-magnitude diagrams showing the distribution of stars selected as potential calibrators (diam OK) and of stars rejected (diam KO). The effect of the interstellar absorption is indicated by the unit vector for $A(\mathrm{~V})=1 \mathrm{mag}\left(A_{I}=0.506 \mathrm{mag}\right.$ and $\left.E(I-J)=0.229 \mathrm{mag}\right)$.

the diagram color $(I-J)$ - magnitude $I$ of the selected and rejected stars. In this figure, the effect of interstellar reddening clearly appears for the stars with a galactic latitude $\leq 20 \mathrm{deg}$. Thus, the stars with strong reddening seem to be automatically rejected from the list of the potential calibrators. This is also a confirmation that one can neglect the effect of interstellar absorption for other stars.

We have described the adaptations and evolutions of the SearchCal to find faint calibrators up to $K \sim 15$ for long baseline interferometric observations. As for the bright-object case, 
Table 6. Positions of the zones used to test the results of the SearchCal request.

\begin{tabular}{ccccc}
\hline \hline $\mathrm{N}$ & Glon & Glat & DiamOK & DiamKO \\
\hline 1 & 165.67 & -24.05 & 116 & 23 \\
2 & 172.10 & -51.93 & 47 & 8 \\
3 & 197.11 & -10.25 & 231 & 40 \\
4 & 206.93 & -16.64 & 31 & 6 \\
5 & 250.53 & -36.00 & 84 & 10 \\
6 & 272.37 & +63.15 & 39 & 7 \\
7 & 278.68 & +22.95 & 156 & 12 \\
8 & 279.91 & -33.45 & 263 & 316 \\
9 & 283.20 & -7.00 & 833 & 41 \\
10 & 301.90 & -44.65 & 505 & 236 \\
11 & 302.07 & -44.95 & 392 & 126 \\
12 & 302.99 & -0.27 & 409 & 394 \\
13 & 306.51 & -2.03 & 688 & 134 \\
14 & 338.21 & +8.92 & 833 & 69 \\
15 & 352.37 & +17.28 & 173 & 92 \\
16 & 359.87 & -17.71 & 137 & 68 \\
\hline
\end{tabular}

we used a dynamical approach, based on an online CDS request and on a dedicated computing program. For the CDS search, the main difference is in the choice of the 2MASS and DENIS catalogs which allow to reach the suitable range of near-infrared magnitudes. Consequently, new surface-brightness relationships were established to compute the angular diameters using near infrared magnitudes and color indices.

For faint stars, the effect of interstellar absorption on the computation of the angular diameter cannot be corrected because the distance as well as the luminosity class are generally unknown. Simulations of this effect have shown that the errors introduced in the computed visibilities are small enough up to baselines of $200 \mathrm{~m}$ so that the large-scale interstellar absorption can be neglected in almost all cases.

Acknowledgements. This research has made use of the Simbad database, operated at the Centre de Données Astronomiques de Strasbourg (CDS), France $^{1}$. This work is supported and funded by the GDR 2596 "Centre Jean-Marie Mariotti" (JMMC) of the $\mathrm{CNRS}^{2}$. This research has made use of the TOPCAT http://www.starlink.ac.uk/topcat/ and STILTS http://www.starlink.ac.uk/stilts/ software, provided by Mark Taylor of Bristol University, England. Thanks to the anonymous referee who provided pertinent advice that helped to improve this paper.

\section{References}

Andersen, J. 1991, A\&AR, 3, 91

Barnes, T. G., Evans, D. S., \& Moffett, T. J. 1978, MNRAS, 183, 285

Bessell, M. S. 1979, PASP, 91, 589

Bessell, M. S. 1983, PASP, 95, 480

Bessell, M. S., \& Brett, J. M. 1988, PASP, 100, 1134

Bonneau, D., Clausse, J., Delfosse, X., et al. 2006, A\&A, 456, 789

Carpenter, J. M. 2001, AJ, 121, 2851

Cutri, R. M., Skrutskie, M. F., van Dyk, S., et al. 2003, 2MASS All Sky Catalog of point sources, ed. R. M. Cutri, M. F. Skrutskie, S. van Dyk, et al.

Delfosse, X., \& Bonneau, D. 2004, in SF2A-2004: Semaine de l'Astrophysique Francaise, ed. F. Combes, D. Barret, T. Contini, F. Meynadier, \& L. Pagani, 181

Delplancke, F., Derie, F., Lévêque, S., et al. 2006, in SPIE Conf. Ser., 6268

Ducati, J. R. 2002, VizieR Online Data Catalog, 2237

Fitzpatrick, E. L. 1999, PASP, 111, 63

Glass, I. S. 1975, MNRAS, 171, 19P

Hakkila, J., Myers, J. M., Stidham, B. J., \& Hartmann, D. H. 1997, AJ, 114, 2043 Johnson, H. L. 1966, ARA\&A, 4, 193

Kervella, P., \& Fouqué, P. 2008, A\&A, 491, 855

Kharchenko, N. V. 2001, Kinematika i Fizika Nebesnykh Tel, 17, 409

Leggett, S. K. 1992, ApJS, 82, 351

Marshall, D. J., Robin, A. C., Reylé, C., Schultheis, M., \& Picaud, S. 2006, A\&A, 453, 635

Mérand, A., Bordé, P., \& Coudé du Foresto, V. 2005, A\&A, 433, 1155

Monet, D. G., Levine, S. E., Canzian, B., et al. 2003, AJ, 125, 984

Mozurkewich, D., Armstrong, J. T., Hindsley, R. B., et al. 2003, AJ, 126, 2502

Ochsenbein, F., Bauer, P., \& Marcout, J. 2000, A\&AS, 143, 23

Perryman, M. A. C. 1997, in Hipparcos - Venice '97, ed. R. M. Bonnet, E. Høg, P. L. Bernacca, et al., ESA SP-402, 1

Petrov, R. G., Malbet, F., Weigelt, G., et al. 2007, A\&A, 464, 1

Richichi, A., Percheron, I., \& Khristoforova, M. 2005, A\&A, 431, 773

Robin, A. C., Reylé, C., Derrière, S., \& Picaud, S. 2003, A\&A, 409, 523

Schmidt-Kaler, T. 1982, Stars and Star Clusters in Landolt-Bornstein New Series Group. 6, Vol. 2b, ed. K. Schaifers, \& H.-H. Voigt

Ségransan, D., Kervella, P., Forveille, T., \& Queloz, D. 2003, A\&A, 397, L5

Skrutskie, M. F., Cutri, R. M., Stiening, R., et al. 2003, VizieR Online Data Catalog, 7233

The, P. S., Thomas, D., Christensen, C. G., \& Westerlund, B. E. 1990, PASP, 102,565

The Consortium Denis 2005, VizieR Online Data Catalog, 2263

van Belle, G. T., \& van Belle, G. 2005, PASP, 117, 1263

Wegner, W. 1994, MNRAS, 270, 229

\footnotetext{
1 Available at http://cdsweb.u-strasbg.fr/

2 Available at http://www.jmmc.fr
} 